\title{
Telemonitoring in patients with chronic respiratory insufficiency: expectations deluded?
}

\author{
Michele Vitacca
}

Chronic respiratory insufficiency represents the advanced stage of a heterogeneous group of respiratory diseases, including COPD, which have an associated high cost burden. ${ }^{1}$ Modern information communication technologies offer new options to deliver remote specialised healthcare, among which telemonitoring, a complex intervention that includes both the electronic transmission of patient information to the healthcare system and the follow-up response by a healthcare professional. The rationale for telemonitoring development in patients presenting with chronic respiratory insufficiency with or without home mechanical ventilation need is related to: (1) progressive ageing of the patient population carrying with it an increased burden of care at home; (2) technological advances; (3) increased healthcare consumption and the need to cut costs; (4) the increasing number of home mechanical ventilation patients across Europe; ${ }^{2}$ (5) difficulties associated with hospital discharge, with tremendous physical and psychological burden for caregivers of home mechanical ventilation patients; ${ }^{3}$ and (6) the opportunity that telemonitoring offers of early remote detection of signs and symptoms of chronic respiratory insufficiency decompensation, ${ }^{4}$ and at-distance tailoring and monitoring of mechanical ventilation and education reinforcement for the patient and caregiver. Given the cost of telemonitoring in terms of human resources, equipment and patients' time, strong evidence of its costeffectiveness is required, in particular as regards the impact of an earlier detection of relapses of chronic conditions. Unfortunately, decision makers, for example, the healthcare authorities, are rushing to introduce telemonitoring in response to the pressure to reduce hospitalisations among patients with chronic diseases, without first carefully weighing up all the evidence. In the last decade, several studies focusing on the effects of various tele-management programmes for

Correspondence to Dr Michele Vitacca, Respiratory Rehabilitation Division, Fondazione Salvatore Maugeri, Via Giuseppe Mazzini, 129, Lumezzane (Brescia)

25066, Italy; michele.vitacca@fsm.it patients with chronic respiratory insufficiency (with COPD being the main diagnosis) have been published. ${ }^{5-8}$

A few years ago, Wooton ${ }^{9}$ concluded that the evidence base for telemedicine in managing chronic diseases is, at present, weak and contradictory. The conflicting results could be due to the fact that to date the literature on telemonitoring in patients with chronic respiratory insufficiency consists mainly of single-centre experiences carried out in small patient cohorts with different diseases, different levels of disease severity and followed up only in the short term; the studies, besides, feature differences of setting, rationale, definition of control group (ie, usual care) and methodology. To date, the evidence as to whether telemonitoring is really effective in patients with chronic respiratory insufficiency seems inconclusive. The 'one glove fits all' approach in offering telemonitoring for chronic respiratory insufficiency seems to be too simplistic for a heterogeneous population such as these patients. Factors that would be important for the successful implementation of telemonitoring are an individually tailored approach, flexibility and a service that is locally responsive.

In this issue of Thorax, Chatwin et al ${ }^{10}$ reported a randomised crossover trial with 6 months of standard best practice clinical care (control) and 6 months with the addition of telemonitoring, in which 68 patients with chronic respiratory insufficiency (38 with COPD) with or without home mechanical ventilation received telemonitoring (a second-generation system) via a broadband link to a hospital-based care team. The composite primary end point was time to first hospital admission for an acute exacerbation. Secondary outcome measures were number of hospital admissions, general practitioner consultations and home visits by nurses, quality of life, hospital anxiety and depression, and self-efficacy score. The main results of the study were: (1) no significant difference in the primary end point between the two groups, (2) hospital admission rate and home visits increased in the telemonitoring group, (3) no change in the number of general practitioner consultations, (4) self-efficacy fell during telemonitoring, while the anxiety and depression score improved marginally. This trial adds new fuel to the debate on optimal disease management for patients with chronic respiratory insufficiency.

There are a number of possible explanations why the telemonitoring approach may not be superior to standard management carried out at home, which may be synthesised as follows:

1. The standardisation of chronic respiratory insufficiency management at home plays a significant role independently of the modality and setting of care and should be considered a primary goal of any care strategyincluding the use of telemonitoringin this patient population.

2. The patients who may benefit most from telemonitoring have not yet been identified. In fact, it is not clear which patients would benefit from specific types of care delivery and, more importantly, what preferences patients have. Although many studies have included patients with severe disease, they vary in terms of the inclusion and exclusion criteria regarding baseline diagnosis, history of exacerbations, previous use of healthcare services such as home visits, hospitalisations, or rehabilitation, as well as requirements for supplemental oxygen or home mechanical ventilation. Patients with severe symptoms, frequent exacerbations, multimorbidity and limited community support might well benefit from telemonitoring. In the case of patients with multimorbidity and very severe disease, telemonitoring needs to be able to deal flexibly with all the conditions that impact on the patient's well-being. The crucial factor in preventing a hospital admission or improving the patient's quality of life may be relief of the distress symptoms due to the different comorbidities rather than an increased monitoring of the chronic respiratory disease. Real life studies on telemonitoring use could help clarify this issue, first, by highlighting the subgroups of patients in whom telemonitoring is most used (and thus presumably of most benefit) and those for whom it is of little advantage, so that the health authorities can adjust their inclusion criteria accordingly, and, second, through classifying patients according to the level of need so that different levels of telemonitoring prescription (more or less technology and/or type of personnel) 
and duration (h/day, months of follow-up) can be allocated to different categories of patients: that is, a more aggressive service for exacerbators and other major users of healthcare, while a less aggressive service for the others. Otherwise, while it is tempting to assume that in the more severe patients their clinical condition is such that some hospital admissions may be inevitable, people with less severe disease might be better targets for telemonitoring to reduce unnecessary hospitalisations. In any case, telemonitoring has not the aim to avoid hospitalisation per se but rather to control the progression of the disease, which sometimes in fact will mean accelerating hospitalisation, face-to-face visits or home care visits to prevent the catastrophic clinical worsening, and subsequent need for intensive care unit admission or mechanical ventilation.

3. The absence of standardised interventions, such as controlled trials with a minimum of 1 year of follow-up, that include a cost-benefit analysis makes it impossible to be confident about the role of telemonitoring in the overall care of patients with chronic respiratory insufficiency.

4. The use of different generations of telemonitoring and e-health devices and platforms may determine substantial differences in the findings across studies. Available telemonitoring devices range from basic firstgeneration systems to the far more complete third-generation systems. First-generation systems allow nonreactive data collection with measurements transferred to the care provider asynchronously; second-generation systems, on the other hand, are equipped with a non-immediate analytical or decision-making structure with synchronous data transfer regulated by automated algorithms in which care providers can recognise important changes; however, delays can occur if the systems are only active during office hours. Third-generation systems are the most complete, and provide constant analytical and decisionmaking support where monitoring centres are led by a physician, staffed by specialist nurses, and have full therapeutic authority $24 \mathrm{~h} /$ day, 7 days/week. The role of the case manager/care manager during telemonitoring use may also vary among different countries depending on the current policy of each country's health system.
5. To evaluate the real cost-effectiveness of new methods such as telemonitoring in this population it is important to understand what is meant by 'standard care' and 'usual care' in the papers so far published. However, standard care varies greatly between European countries, and within each country. ${ }^{11}$ Some studies have also proposed health economic assessments ${ }^{5} \quad 6 \quad 12$ with non-definitive conclusions. For example, in Chatwin et al's ${ }^{10}$ study the standard care was applied according to the local guidelines with a personalised home care plan for escalating therapy including instructions regarding antibiotic therapy, corticosteroid treatment and adjustment of inhaled medication. Patients were provided with the phone number of a contact person in their medical team and also had ready access to respiratory care nurses who were part of the trial, as well as to their general practitioner. Unfortunately, this 'standard' care is not a common or mandatory care approach in all EU countries. If an extensive home care package with strong community links exists, telemonitoring may add little additional benefit, whereas in the trials with less community support telemonitoring seems to show more benefit in terms of team expertise, and the patient's (or carer's) self-efficacy. Now, the question to evaluate is if the superiority of telemonitoring to the gold standard is really the goal. Equivalence between telemonitoring and the gold standard may be a more appropriate goal; indeed, an intervention that costeffectively improves a suboptimal service bringing it on a par with the gold standard would be a success. Cost effectiveness could be the 'gold standard' for each new health service. It is not important for each health organisation to push for a 'unique modality' of continuity of care but to press for the 'most efficient' one respecting shared and standardised clinical and scientific targets for chronicity care.

6. Last but not least, negative or positive results clearly depend on the expected outcomes of the study (eg, health use, patient related outcomes, adherence, mechanical ventilation initiation and adaptation, need for palliation care) and corresponding methodological development, which differ from one study to the next.

As future directions, more attention needs to be focused on how to accommodate the increasing number of patients with chronic respiratory insufficiency in a postdischarge telemonitoring management programme with real integration between hospital and primary care professionals according to quality standards. The self-management support must also become more integrated, with standardised decision support and outcome measures plus electronic information so that critical information is shared among the various health professionals involved in the home programmes. In addition, more research is required on the organisational implications of introducing telemonitoring to avoid that a new service may duplicate a traditional system with more inefficiency and costs increase, on the security and confidentiality of patient data, on the responsibilities and potential obligations of health professionals and on EU jurisdictional problems regarding e-health systems. Finally, we need to provide a useful benchmarking picture of different telemonitoring good practices around Europe as an aid to those who fund telemonitoring services in their decisions as regards personnel investment, reduction of redundancy and duplication of care services, as well as prioritisation of services.

In conclusion, at the moment the fundamental prerequisite for the efficacy of telemonitoring in chronic respiratory insufficiency management is to establish common standardised protocols rather than determine how to deliver the care. The absence of conclusive evidence for the benefit of telemonitoring in chronic respiratory insufficiency should, however, not be taken as evidence of an absence of benefit. It is clear that telemonitoring alone is not sufficient in itself to yield a better outcome; telemonitoring could be a key element in management of patients with chronic respiratory insufficiency, but it is difficult to evaluate its benefit without considering the other services received by patients (home care, access to hospital, social care). Considering the overall care 'package' received by the patient, telemonitoring may be included as one of the services offered within the package. But other aspectsquality improvement, integration of programmes and services, increase of collaboration and communication across the different care settings and the development of a shared vision, goals and priorities-are needed to improve the efficiency of the healthcare services provided for chronic patients. Successful implementation of telemonitoring can change how things are done and, in turn, the configuration of services.

The key point in optimising the use of telemonitoring is to correctly identify who the ideal candidates are, and at what 
time they should receive telemonitoring and for how long.

In other words, oscillating between expectations and disillusionment, the current dilemma is not 'telemonitoringyes or no?', but how to use it in a mature and balanced manner in such a way as to enhance the health outcomes for our chronic patients.

Competing interests None declared.

Provenance and peer review Commissioned; externally peer reviewed.

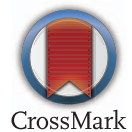

To cite Vitacca M. Thorax 2016;71:299-301.

\section{SLinked}

http://dx.doi.org/10.1136/thoraxjnl-2015-207045
Thorax 2016;71:299-301.

doi:10.1136/thoraxjnl-2015-208211

\section{REFERENCES}

1 Perera PN, Armstrong EP, Sherrill DL, et al. Acute exacerbations of COPD in the United States: inpatient burden and predictors of costs and mortality. COPD 2012;9:131-41.

2 Lloyd-Owen SJ, Donaldson GC, Ambrosino N, et al. Patterns of home mechanical ventilation use in Europe: results from the Eurovent survey. Eur Respir J 2005;25:1025-31.

3 Vitacca M, Grassi M, Barbano L, et al. Last 3 months of life in home ventilated patients: the family perception. Eur Respir J 2010;35:1064-71.

4 Borel JC, Pelletier J, Taleux N, et al. Parameters recorded by software of non-invasive ventilators predict COPD exacerbation: a proof-of-concept study. Thorax 2015;70:284-5.

5 Vitacca M, Bianchi L, Guerra A, et al. Tele-assistance in chronic respiratory failure patients: a randomised clinical trial. Eur Respir J 2009:33:411-18.

6 Cartwright M, Hirani SP, Rixon L, et al, Whole Systems Demonstrator Evaluation Team. Effect of telehealth on quality of life and psychological outcomes over 12 months (Whole Systems Demonstrator telehealth questionnaire study): nested study of patient reported outcomes in a pragmatic, cluster randomised controlled trial. BMJ 2013;346: f653.

7 Pinnock $\mathrm{H}$, Hanley J, McCloughan L, et al. Effectiveness of telemonitoring integrated into existing clinical services on hospital admission for exacerbation of chronic obstructive pulmonary disease: researcher blind, multicentre, randomised controlled trial. BMJ 2013;347:f6070.

8 McLean S, Nurmatov U, Liu JL, et al. Telehealthcare for chronic obstructive pulmonary disease: Cochrane Review and meta-analysis. Br J Gen Pract. 2012;62: e739-49.

9 Wootton R. Twenty years of telemedicine in chronic disease management-an evidence synthesis. J Telemed Telecare 2012:18:211-20.

10 Chatwin M, Hawkins G, Panicchia L, et al. Randomised crossover trial of telemonitoring in chronic respiratory patients (TeleCRAFT trial). Thorax 2016:71:305-11.

11 European Commission. Commission staff working document on the applicability of the existing EU legal framework to telemedicine services. Brussels, 7 Dec 2012.

12 Stoddart A, van der Pol M, Pinnock $\mathrm{H}$, et al. Telemonitoring for chronic obstructive pulmonary disease: a cost and cost-utility analysis of a randomised controlled trial. J Telemed Telecare 2015:21:108-18. 